\title{
Berichtigungen zu 0. Schultze's jüngstem Aufsatz über die Bedeutung der Schwerkraft für die Entwickelung des thierischen Embryo und Anderes \%.
}

Von

W. Roux.

Der Autor versucht zunächst meine in Band IX pag. 479-499 dieses Archivs gegebene Widerlegung seiner theoretischen Auffassungen uber die Wirkung der Schwerkraft zuruckzuweisen.

Er giebt daher im ersten Theil seiner Abhandlung eine zusammenhängende und zwar klar verständliche Darlegung seiner Auffassung, aus der jetzt jeder Leser sich selber ein Urtheil bilden kann. Unsere Hauptdifferenz beruht danach aber noch ganz wie bisher darauf, dass Schultze schließt: weil abnorme Wirkungen der Schwerkraft schädlich für die Entwickelung sein können, sind die normalen Wirkungen der Schwerkraft zur normalen Entwickelung nöthig; während ich schließe, dass bloß die Fernhaltung der abnormen Wirkungen der Schwerkraft nöthig ist. Darüber brauchen wir kein Wort mehr zu verlieren. Ebenso sehe ich ron allerhand kleinen Unrichtigkeiten ab, wie sie bei Polemiken leicht sich einzustellen pflegen, um hier bloß einige Punkte, auf die ich mehr Werth lege, zu behandeln.

Die typische Anordnung der Dottermassen im Eierstocksei bleibt nicht, wie Schultze (pag. 311) angiebt, trotz der Umordnnngstendenz der Schwerkraft ganz erhalten; sondern ich habe an schwimmend gemachten Eiern von Rana fusea beobachtet, dass die unbefruchteten Eier sich mit ihrer Eiachse in verschiedener Weise schief einstellen, und dass erst nach der Besamung die typische senkrechte Einstellung sich herstellt, was also auf vorher bestehende $A$ bweichung der Dottermassen von der "typischen Anordnung" hinweist.

1) Archiv f. mikr. Anat. Bd. 56. pag. 309-334. 
Auf pag. 313 sagt Schultze: $\gg$ Es würde keine Frösche mehr geben, wenn nicht die Eier drehbar in der Hülle wären, denn die abnorm angreifende Schwerkraft würde schonungslos die vorgebildete Anordnung der Eisubstanzen zerstören. « Das ist nicht zutreffend, denn erstens wiirden manche Eier zufällig mit den Eiachsen senkrecht stehen, und außerdem aber wissen wir, dass zwangsweise Sehiefstellung von z. B. $40^{\circ}$ gar nichts schadet.

Anzuerkennen ist, dass Schultze jetzt Eier, die in so starkem Zwang gehalten sind, dass sie sich erst im Laufe von 11-14 Stunden nach der Umkehrung wieder ganz aufwärts drehen konnten, als in unvollkommener Zwangslage befindlich bezeichnet, während er rorher, um meine bezüglichen Angaben als unrichtig zu markiren, diese hochgradige Fixation stets "keine Zwangslage" nannte.

Von einer »A ufhebung der Wirkung der Schwerkraft" dureh meine Rotationsversuche, wie O. Schultze mir pag. $322 \mathrm{zu}-$ schreibt, habe ich natülich nie gesprochen. Man wird oft "Aufhebung der richtenden Wirkung der Schwerkraft " erwähnt finden; und wenn, wie mir aber nicht bekannt ist, einmal dies Beiwort fehlen sollte, so wird aus dem Zusammenhang unfehlbar klar, dass nur die richtende, orduende Wirkung als aufgehoben gemeint ist.

Schulrze theilt jetzt, meinen Bericht uber seine Ansichten ergänzend, mit, dass er sich 1897 in Gent dahin auggesprochen hat: "dass er von einer anderen als (NB. gleich mir von) der richtenden Wirkung der Sehwerkraft nicht gesprochen hatc. Es wäre zu empfehlen gewesen, dass er dies Wesentliche auch in seiner ausführlichen Abhandlung erwähnt bätte; denn nach seinen Vorstellungen von der absoluten Nothwendigkeit der Schwerkraft zur Entwickelung konnte man wohl allerhand Räthselhaftes vermuthen. Dass mein, wie er sagt, "schlechter " Rotationsapparat geschlottert hat, ist bei diesem Versuche gerade ein Vorzug vor seinem ruhiggehenden * Klinostaten *. Denn wenn, nach Schultze's Annahme, bei den Umdrehungen des ersteren Apparates wirklich »die Eiachsen gleichsam durch einander kollerten «, so verhinderte das die von ihm bemängelte Einstellung der Eiachsen in die Rotationsebene, welche übrigens für unsere Zwecke ohne Belang wäre.

Schultze kommt meiner Auffassung einen Schritt entgegen, indem er zugiebt, dass "permanente Normaleinstellung" (und NB. zwar stundenlang) nicht nöthig ist.

Auch schließt er sich jetzt, aber mit anderen Worten (pag. 324), meiner Auffassung an, dass bei meinen Rotationsversuchen wegen der 
in jedem Noment wechselnden Richtung die Schwerkraft nicht Zeit hat, die verschieden schweren Dottertheile umzuordnen. Das war die Grundlage meiner im Jahre 1894 angestellten Versuche und ihrer Verwerthung.

Ferner nähert sich seine Zusammenfassung ein wesentliches Stiick meiner wohl genïgend bekannten Auffassung; indem er sagt (pag. 326):

* Die in der Richtung der Eiachse wirkende, die normale Struktur des befruchteten Eies, allein' sicher erhaltende Schwerkraft ist so lange fuir das Ei entbehrlich, als die in Folge ihrer A usschaltung eintretende abnorme Schwerkraftwirkung keine Zeit gervinnt, die normale Anordnung der Substanzen von verschicdenem specifischen Gewicht zu zerstören."

In diesem Satze habe ich diejenigen Stellen, welche ich noch nicht fuir richtig halte, gesperrt drucken lassen. Denn die in der Richtung der Eiachse wirkende Schwerkraft ist nicht die "die normale Struktur des befruchteten Eies allein sicher erhaltende Kraft«, sondern, sofern störende Einwirkungen nicht stattfinden, erhiilt sich diese Struktur des Froscheies von selber, schon nach dem Gesetz der Trägheit. Und da sie sich auch bei geringen störenden Einwirkungen wie Schiefstellungen von weuigen Graden, Wackeln der Eier und bei meiner Rotation mit je einer Umdrehung in etwa 2 Minuten erhält, so müssen auch noch die Anordnung direkt erhaltende Ursachen im Eie selber vorhanden sein nud zur Wirkung gelangen.

Bei stark schief geneigter Zwangslage aber reichen, wie Bonx's Untersuchungen ${ }^{1}$ ) erkennen lassen, diese die Anordnung erhaltenden Kräfte gegeniiber den umordnenden Wirkungen der Schwerkraft nicht mehr ans; ebenso bei den später von Schultze angewandten, iiberaus langsamen Umdrehungen; denn die Eier verfärbten sich dabei grau (ein Beweis, dass die hellen und dunklen Dottersubstanzen günzlich durch einander kamen) und die Eier starben ab.

Obschon ferner die Eier ron hana esculenta sich mit ihrer Eiachse nicht senkrecht, sondern etwa $40^{\circ}$ schief zur Richtung der Schwerkraft einstellen, formulirt Schultze doch (pag. 326) den folgenden, diesem Verhalten direkt widersprechenden Satz:

»Es hat noch Niemand etwas vorgebracht gegen meine Beweis-.

1) Born, G., Über den Einfluss der Schrere auf das Froschei. Archiv t. mikr. Anat. Bd. 24. 1885. 
fiihrung, dass für die normale Entwickelung überall da, wo sich unter Einfluss der Schwerkraft Froscheier entwickeln, die Schwerkraft mit der Eiachse einen Winkel von $0^{\circ}$ bilden muss, weil unter allen anderen Winkelstellungen zwischen $0^{\circ}$ und $180^{\circ}$ Missbildungen, der Größe des Winkels proportional, entstehen bezw. der Tod eintritt, oder dass, mit anderen Worten, der normale Schwerkrafteinfluss für das Ei nöthig ist. Sollte es aber Jemand unternehmen, so sehe er sich vor, dass er nicht gleichzeitig das fundamentale Princip experimenteller Forschung iuberhaupt verlache. *

Von dieser somit ganz irrthümlichen Ausführung interessirt uns hier mur noch das Wort pnormale "Entwickelung. Schubrze betritt dadurch mit einem Fuße schon die Brücke, die ich ihm in meiner rorigen Schrift am Schlusse gebant habe, indem ich (pag. 433) darauf hinwies, dass er nuu vielleicht meine bisher von ihm ignorirte Unterscheidung einer typischen oder normalen und einer atypischen oder regulatorischen Entwickelung acceptiren und sagen werde: nur bei normaler Schwerkraftwirkung finde die normale Entwickelung statt, anderenfalls regulatorische Entwickelung. So spricht er an anderer Stelle auch bereits einmal von der regulatorischen Entwickelung.

Aber der Satz bleibt doch selbst auch in dieser Beziehung unzutreffend; denu trotz der fehlenden normalen Schwerkraftwirkung entwickelten sich meine rotirenden Eier auf normalem Wege; es war nichts Abnormes während des Verlaufs der Entwickelung zu bemerken, sie furchten sich auch ganz normal (Ges. Abh. II. pag. 269).

Auch entstehen in Folge abnormer Schiefstellung der Eiachse geringen Grades nicht nothwendig Missbildungen, wie Schclize behauptet. Man sieht sogar direkt, dass die gestörte Anordnung unter der von mir beobachteten nachträglichen, symmetrisch zur ersten Furche erfolgenden Umordnung des Pigments der Eirinde wieder hergestellt wird (Ges. Abh. II. pag. 328). Das ist regulatorische Entwickelung, aber keine Missbildung.

Ferner berichtet Schultze, ich hätte nach meiner Angabe schon 10 Jahre vor ihm versucht, durch Umkehrung von Froscheiern nach der ersten Furchung "Doppelbildungen zu erzielen

Das habe ich ebenso wenig "zu erzielen« versucht, wie O. Schultze es gethan hat. Denn dass bei Umkehrung der Eier Doppelbildungen entstehen wiirden (oder dass nach Schulrze's damaliger Vorstellung von der Ursache der Doppelbildungen: sich dabei der Schwerpunkt theilen werde), vermochte Niemand zu ahnen. 
Ich hatte aber 10 Jahre vor Schultze in Pflüger's Zwangslage gehaltene Eier nach der ersten Furchung umgedreht, bloB um zu sehen, was daraus werde, aber nichts besonders Bemerkenswerthes danach erhalten. Schultze hat später die Umkehrung der Eier gleichfalls in dieser Phase vorgenommen, ohne von meinem Versuch etwas zu wissen; er fixirte die Eier jedoch durch Pressung zwischen wagrechte Platten und erhielt danach das überraschende Ergebnis.

Nach seinem Bericht in Straßburg machte ich Schultze's Versuch nach, also natiurlich mit Plattenpressung, wie ich Ges. Abh. II. pag. 936 und in meiner letzten Abhandlung (dieses Archiv. Bd. IX. pag. 484) genuigend deutlich angegeben habe; daneben zum Vergleich wurden auch wieder, aber nicht allein, wie Schultze berichtet, in PfuÜGER's Zwangslage gehaltene Eier umgedreht. In meiner Abhandlung über die Entwickelung isolirter erster Blastomeren (dieses Archiv. Bd. I. pag. 598) findet sich aber in der That eine Angabe, welche so gefasst ist, dass sie den Leser zu dieser Annahme reranlassen konnte oder musste; und diese Stelle hatte Schultze wohl noch in der Erinnerung. Ich hätte dort der Mittheilung: „In Folge des Berichts Schultze's wiederholte ich diesen früheren Versuch (NB. der Umkehrung der Eier in PrLüger's Zwangslage) im Juni 1894 an 45 Eiern« - die Worte: »nun aber natiirlich nach Schurtze mit Zwang durch Plattenpressung * hinzufügen müssen. Ich habe damals (1894) mit großer Erwartung die Doppelplatten mit den zwischen ihnen liegenden platt gepressten Eiern von oben und unten beobachtet, letzteres indem ich sie, um die Umdrehung zu vermeiden, über den Kopf hielt. Da aber keine Doppelbildungen, sondern nur Halbbildungen und ganz todte Eier entstanden und da ich in der verspäteten Zeit der Laichperiode eine genügende Ursache für das abweichende Resultat erkannte, so begnügte ich mich mit dem kurzen, auf pag. 936 des zweiten Bandes meiner gesammelten Abhandlungen gegebenen Bericht über diesen Versuch.

Was die von Schulcze beregte Art der Polemik angeht, so darf ich wohl darauf hinweisen, dass ich, von einem einzigen Male, wo ich direkt einen thatsächlichen Irrthum sogleich bewiesen habe (s. u. pag. $254 \mathrm{Nr}$. 3), abgesehen, nie thatsächliche Angaben eines Autors als solche in $Z$ weifel gezogen habe, sondern dass ich nur die Umstïnde, unter denen sie gewonnen worden sind, sowie die den Thatsachen gegebenen Deutungen oder die aus ihnen abgeleiteten Folgerungen bemängelt habe. Anch verschweige ich meinen Lesern nie die guten Argumente des Gegners. 
0. Schultze dagegen sowie 0. Hertwig, auf den er sich zu stiitzen sucht, vermochten sich in ihrer Polemik mit mir sehr oft nicht anders als mit der stereotypen Wendung zu helfen: diese Thatsache Rocx's existirt nicht, oder dadurch, dass sie ihren Lesern diejenigen Thatsachen und Argumente, die sie nicht widerlegen konnten, ganz verschwiegen. Beides habe ich ihnen schon oft vorzuhalten Veranlassung gebabt.

Wirklich falsche thatsächliche Angaben werden aber nur sehr selten als die Ursache von wissenschaftlichen Differenzen ermittelt; meist handelt es sich nur um die ron mir erwähnten Irrthümer. Die genannten Autoren und manche Andere unterscheiden bei der leichtfertigen Zuruickweisung thatsächlicher Angaben nicht einmal zwischen solehen Thatsachen, die, sofern sie bestehen, leicht und sicher zu beobachten sind, wie z. B. die früher ron 0 . Hentwig verneinte Existenz der Hemiembryonen des Frosches oder die von O. Schulitze bisher abgewiesene Rückenlage von Froschembryonen, Thatsachen, deren unrichtige Angabe auf gröbster Leichtfertigkeit beruhen müsste, so dass die Verneinung einer derartigen Angabe überhaupt nur nach vollkommen sicherem Nachweis ihrer Unrichtigkeit geschehen dürfte.

Außer solchen giebt es aber viele andere Thatsachen, welche an sich sehr schwer zu beobachten sind, da ihre sie von bezuglichen anderen Thatsachen unterscheidenden Merkmale sehr feine oder auch fluktuirende und an der Grenze der Leistungsfähigkeit der angewandten Methode liegende sind, so dass man in Bezug auf sie ohne beleidigende Wirkung mit der Möglichkeit eines Irrthums des Gegners rechnen darf.

Wenn ferner eine Thatsache in neue Beziebungen gerïckt wird oder eine andere Ableitung erfährt, dann kommen plötzlich sekundäre oder tertiäre feine Charaktere zur Geltung, die früher vielleicht als nnwesentlich nicht oder nicht genügend beachtet und geprüft worden sind. Der Autor, der diese neue Auffassung vertritt, hat dann aber alle diese unterscheidenden Merkmale genau darzulegen, muss berichten, dass er sie in natura geprüft und die seine Auffassung beweisenden Merkmale vollständig vorgefunden hat.

Das hätte 0 . Schultze thun muissen, wenn er beweisen wollte, dass die von ihm früher verneinte und nun endlich gesehene Lagerung des Froschembryo mit dem Rücken nach unten nicht, wie ich abgeleitet habe, durch Überwachsung, durch Epibolie der unteren Eihälfte, sondern durch Drehang des ganzen Eies hervorgebracht wird. 
Aber auch hier fehlt wieder das Wesentliche, fehlt dieses Hauptkapitel in seiner Arbeit, es ist bloß dureh ein Paar unzureichende Bemerkungen vertreten. Zudem beweist die ron Schucrze als Hanptargument erwähnte weiße Beschaffenheit des Dotterpfropfes (pag. 330) an sich gar nichts gegen die Wanderung des Urmundes um 170', denn der Dotter ändert seine manchmal graue Farbe auch sonst nicht selten lokal, sowohl vor wie während der Gastrulation; und das Übersehen derartiger Ïuderung der Pigmentvertheilung auf früherer Stufe war die Ursache der irrthümlichen Behauptung: Schultze's, bei Rana fusca stände die Eiachse $45^{\circ}$ schief'j.

Auch habe ich bereits bei der Verwerfung der oberflächlichen Pigmentfleckenmarken 0 . Schultzr's mitgetheilt, dass die Pigmentvertheilung in der Eioberflache sehr verïnderlich ist, dass Pigmentanhäufung und Pigmentschwund an umschriebenen Stellen der Blastula zur Beobachtung kommt ${ }^{2}$. Und an den einzelnen Zellen habe ich das Gleiche dargethan ${ }^{3}$ ).

Zudem ist die Hanptsache zn bericksichtigen, dass ich meine Anffassung noch durch Anstichversuche goestiitzt habe und dass

1) Etwa um die Nitte der achtziger Jahre hatte ich Eier von Rana fusca, welche anf der Unterseite statt weiß dunkelgrau waren, gleichwohl aber später, sofern ich mich noch recht erinnere, in dem ringförmigen Urmund einen hellen Dotterpfropt darboten.

Ferner kommen bei dieser Species nicht selten Eier vor, deren weißer Pol oder sogen. weiße Hemisphäre sehr klein ist, etwa nur $50^{\circ}$ umfasst, so dass die stets ein wenig unter dem Eiäqutor (nicht aber, wie Schultze sagt, immer am Pigmentrande, also an der urspringlichen Grenze von schwarzem und weißem Pol) erfolgende erste Anlage des Urmundes ganz in Bereiche der ursprïnglichen schwarzen Hemisphäre stattfindet. Daher müsste der Urmund sehr schwer zu sehen sein. Gleichwohl findet man ihn nach der Umkehrung einer Schale, so viel ich mich erinnere, leicht an allen Eiern, weil unuittelbar unterhalb der stets schwarzen dorsalen Urmundslippe die anstoßenden Dotterzellen eine Aufhellung erfahren haben.

Diese Verhältnisse mïssen nunmehr aufs Neue geprïft werden, einmal weil ich sie friher nicht beschrieben und jetzt nur aus ziemlich entfernter Erinnerung vorgefiihrt habe, anderen Theils aber, weil sie durch die obige Behauptung O. Schultze's über die Entstehung der Riickenlage des Froschembryo von Bedeutung geworden sind, ohne dass dieser Autor daraus Veranlassung genommen hätte, sein Hauptargunent für die Drehung des Eies: die weiße Farbe des Dotterpfropfes selber auf seine Stichhaltigkeit zu prüfen, bevor er auf Grund desselben ein apodiktisches Urtheil abgab.

2) Biol. Centralbl. ViII. 1858. pag. 408. Anat. Anzeiger. III. 1558, pag. 701 Ges. Abh. Il. pag. วั30.

3) Roux, W., Selbstordnung der Furchungszellen. Dieses Archiv. 1596. Bd. III. pay. 404. 
diese Versuche sowic die aus ibnen gezogenen Folgerungen im rorigen Jahre von BER'TACHIXI ${ }^{1}$ ) durchweg bestätigt worden sind, derart, dass nach diesem Autor die Epibolie sich sogar noch etwas weiter ausdehnt, als ich es angegeben habe. Zugleich ist anf die bezigglichen Experimente Banfurth's an Siredon zu verweisen ${ }^{2}$ ).

Es war ein sehr unglücklicher Zufall, dass Schultze, wie er jetat sagt: rou seinen wahllosen "Versuchen mit Zwangslage wormal gestellter Eier nur einen einzigen Versuch, und zwar gerade den rom 24. März (1897), also von einer Zeit der Verzögerung der Laichung, mittheilte, in der nach meinen Erfahrungen Stehenbleiben auf der Gastrulationsstufe zu den gewöhnlichsten Erscheinungen auch olne Zwangslage gehört. Da er aber "zahllose" Versuche der Art gemacht hat, und da er anßerdem normal, also ohne Zwang, gehaltene Kontrolleier daneben gehalten hat, die, wie er zwar nicht direkt sagt, aber doch dadurch suggerirt, dass sonst der Tersuch für ihn werthlos wäre, sich (NB. trotz der vorgeschrittenen Zeit!) normal entwickelten, und da er auch Kontrolleier in Zwang hielt, welche nicht wie die anderen behufs Prüfung der Vollkommenheit der Zwangslage umgekehrt worden wären, welche sich aber ebenso entwickelten wie die probeweise $11-14$ Stunden umgekehrten Eier, so entfallen die beziiglichen Einwendungen. Dessgleichen entfällt daher mein, übrigens zu seinen Gunsten, nämlich um seine thats:̈ichlichen Angaben nicht bezweifeln zu müssen, gemachter Erklärnusversuch, dass erst durch diese 11-14stindige Umkehr die paradose Drebung der Eier statt aufwïrts nach abwärts veranlasst worden sei.

Wenu Schucitze alle die wesentlichen Cnastände, die zur Beurtheilung jedes dieser Versuche gehörten, im Zusammenhange und vollstïndig mitgetheilt hätte, hätte ich mir die Mühe dieser Deutungen sparen und sogleich die nachstehend folgende Deutung seiner beziiglichen Versuche geben können.

Da durch meine früheren Rotationsversuche zur Genüge bewiesen ist, dass die normale richtende Wirkung der Schwerkraft zur Entwickelung der Froscheier nicht nöthig. ist, so leite ich seinen Befund, dass seine in normaler Stellung aufgesetzten und durch entsprechende

y) Bertachini, P., Morfogenesi e Teratogenesi negli Anfibi anuri. IIa Serie: Blastoporo e organi assili dorsali dell' embrione. Internat. Monatsschr. f. Anat. u. Phys. 1s99. Bd. 16. Heft 11/1?.

7) Barfurth, D., ¿̈ber die organbildenden Keimbezirke und kïnstlichen Hissbildugen des Amphibieneies. Anat. Hefte. 1893. pag. 355-389. 
Trockenhaltung in stärkster, nach Schultze allein rollkommener Zwangslage erhaltenen Eier auf der Gastrulastufe stehen blieben, nicht wie Schultze von der fehlenden normalen Wirkung der Schwerkraft, sondern von den durch die starke Trockenhaltung bedingten Momenten des Luftmangels und zu starker Pressung ab. Der Umstand, dass die Eier nach Wasserzusatz sich weiter entwickelten, ist eher von der Aufhebung dieser Schädlichkeiten abzuleiten als davon, dass die Eier sich nun ein wenig drehen und genau nach der Richtung der Erdachse einstellen konnten. Letztere Begründung ist schon dadurch als unzutreffend charakterisirt, dass bei der nach SCHULtze zwar nur » unvollkommenen " Zwangslage, welche aber bedingt, dass der Embryo mit dem Rücken sich nach unten statt nach oben lagert, die Entwickelung ganz gut verlïuft.

Schultze steht ferner bereits auf dem Punkte, dass er sagt (pag. 327): „Die Eier von Roux aber bildeten das Medullarrohr, was ich als ein sicheres $Z$ eichen nicht rollkommener $Z_{\text {wang }}$ slage erwiesen habe."

Damit läuft die Differenz in dieser Hinsicht auf einen Streit hinaus iiber das, was Schultze vollkommene und unvollkommene Zwangslage nennen will.

Die Frage, ob Schultze's » vollkommene « Zwangslage wirklich die alle in vollkommene Zwangslage ist, während die von mir für vollkommen gehaltene, nach Schultze unvollkommen ist und daher statt durch Epibolie auch durch paradoxe Drehung die Medullarplatte nach unten gelangen lassen kann, ist eine Frage fuir sich, die SCHulTzE, wie wir sahen, leider nicht geniggend geprüft hat, um sein Urtheil begriunden zu können. Neben dieser Frage interessirt uns die angeblich allein vollkommene Zwangslage nur in so fern, als sie vermuthlich das Ei durch Pressen und Luftmangel schaidigt, ohne etwas für die Schwerkraft zu beweisen.

Es ist nach meiner Meinung sehr bedenklich, dass Schlltze annimmt and annehmen muss, Froscheier, welche in so starker, nach ihm früher "keiner", jetzt »unvollkommener" Zwangslage gehalten werden, dass sie dem natürlichen starken Drang zur Aufwärtsdrehung, der dadurch bedingt ist, dass die Medullarplatte aus protoplasmatischen Zellen besteht, die specifisch leichter sind als die Dotterzellen, nicht folgen können, nun aber, weil sie an dieser Drehung verhindert sind, plötzlich die Fähigkeit erlangen, trotz dieses Zwanges sich mit den specifisch leichteren Theilen abwärts zu drehen. 
Obgleich nun nach meiner Auffassung dieser Zwang ausreichend ist. um ebenso wie die Drehung nach aufwärts auch die ron SchCLTze angenommene und nicht erwiesene, jedenfalls aber wohl viel schwächere, Drehungstendenz nach abwärts an der Vollziehung zu hindern, so braucht dieser Zwang damit nicht auch zugleich die Überwachsung der Unterseite zu verhindern, da diese immer nur mit oberflächlichen Materialumlagerungen an cirkumskripter Stelle (vorzugsweise in der Umgebung des Urmondes) vor sich geht, während bei Drehung des Eies in toto, von den Umdrehungspunkten abgesehen, an allen Stellen der Oberfläche zugleich Verschiebung gegen die das $\mathrm{Ei}$ pressende Gallerthülle stattfinden müsste (siehe Roux, Biol. Centralbl. VIII. pag. 409).

Zugleich möchte ich darauf hinweisen, dass aus meiner Annahme der Epibolie durchans nicht zu folgern ist, dass die Überwachsung mit der Innen- and Außenseite der Medullarplatte ganz gleichmai $\mathrm{ig}$ geschehen müsse, dass also dabei nicht etwa durch stärkeres Wachsthum auf der Außenseite äußere Zellen über den Umschlagsrand nach innen gelangen könnten, wie es aus KoPscr's photographischen Aufnahmen des gastrulirenden Froscheies abgeleitet worden ist. Diesen letzteren Vorgang kann man wohl auch als Einstiilpung, Inragination bezeichnen, wenn man eine Vorliebe für dieses Wort hat. Ich habe aber, wie sich ergiebt, das Wort seiner Zeit in anderem Simne gebraucht, als ich sagte, dass die Gastrulation des Froscheies, alogesehen vom ersten Beginne an, der nach meiner Beobachtung eine wirkliche Einstülpung oberflächlicher Zellen in ihre Unterlage hinein darstellt, sowie ron der nachträglichen Ausweitung des platten primären Lrdarmranmes!), nicht durch Einstiilpung, sondern durch Überwachsung der Unterseite stattfindet, also ohne Einsenkung obertlächlicher Zellen in ihre Unterlage ror sich geht. Hier dagegen findet Einwärtslagerung von Zellen über einen eben erst eine freie Fläche bedeckenden Rand statt, was doch etwas Anderes ist.

O. Schubtze hat sich bisher begniigt gehabt, einzelne meiner Thatsachen als nicht existirend zu bezeichnen. In der jetzigen Lage kann er sich nur noch durch einen Massenmord vou thatsächlichen Angaben retten. den wir zur Erheiterung mittheilen und aufbewahren wollen.

1) Biol. Centralbl. VII. pag. 425̃. Roux's Referat über O. Schultze's erste beziigliche Arbeit. 
Er sagt (pag. 333 ; dass niemals ein objektiver, mit dem Objekt genügend vertranter Forscher meine beiden Beiträge 3 und 4 zur Entwickelungsmechanik, die er als rorgefasste Gedanken und nachträgliche oberflächliche Eier- und Spermatozoenspielerei bezeichnet, sowie manche andere meiner Beiträge ernst nehmen wird; und dass auch der Cytotropismus wohl keine Berechtigung auf bleibendes Dasein haben werde.

Zum Verständnis dieser Massenhinrichtung von Thatsachen ist es nöthig, mitzutheilen, dass in diesen Arbeiten Nr. 3 und 4 schon. beror 0 . Schultze aus nicht beweisfähigen deskriptiven Thatsachen die Anlage der Symmetrie des Embryo im noch unbefruchteten Eie ableiten zu können glaubte, durch eine liickenlose Serie rou Versuchen die Unrichtigkeit dieser Auffassung vollständig erwiesen war. Schctuze kanute aber damals meine Versuche wohl nicht genügend, danach vermochte er sie nicht genïgend zu verstehen. ebenso wie es ihn, wohl weil es analytische Versuche sind, anch nicht möglich gewesen ist, sie richtig nachzumachen. Ich bin aber iiberzeugt, dass Letzteres, wenn auch wohl nicht mehr 0. Schultze. so doch manchem anderen Autor mit der Zeit vollkommen gelingen wird.

Schließlich nennt es Scuctrze eine Entstellung, dass ich sagte, er habe eine ganze Reihe meiner Angaben frïher angezweifelt und danach als richtig erkannt. Er erinnert sich selber nur noch daran. dass er behauptet hat, meine (und NB. seines eigenen Vaters) Angabe, dass die Eiachse bei Rana fusea sich senkrecht einstelle, sei falsch. Er nennt das jetzt ein Missrerständnis, obschon es auf Beobachtung'srersüumnis Schcltze's berulte, denn er machte mir Einwendungen gegen meine bestimmt lokalisirte Befruchtung, weil die Eiachse schief stunde. Es handelte sich somit um den Anfang der Entwickelnng, während sich dann herausstellte, dass Scuctize seine Eier uberhaupt erst einige Stunden später beobachtet batre, erst zu einer Zeit, als schon die von mir entdeckte nachträgliche Aufhellung des Dotters auf einer Seite eingetreten war. (Biol. Centralbl. VIII. pag. 402-405.)

Außerdem hat er weiterhin seine Augaben den meinigen entsprechend berichtigt darin:

2) dass die Froscheier einen centralen, feinkörnigen (oft braunen) Dotter haben (s. Biol. Centralbl. VIII. pag. 406);

3) dass die Dotterkörner des Froscheies iiberhaupt nicht, wie er behauptet und abgebildet hatte, in jeder Horizontalebene gleich groß sind (Biol. Centralbl. VII. pag. 4.32 u. VIII. pag. 406); 
4) dass nicht derjenige Punkt des Pigmentrandes, welcher dem rerschwindenden Keimbläschen am nächsten liegt, die Stelle der Anlage des Urmundes bezeichnet, da ich zeigte, dass der Meridian der Furchungsebene nicht durch die Lage der Fovea germinativa bestimmt wird (Biol. Centralbl. VIII. pag. 401);

5) dass oberflichliche Pigmentflecke des Eies nicht als Marken brauchloar sind, weil sie wandern und rerschwinden können (Ges. Abh. II. pag. 533\%. (Schultze verwendet in den letzten Arbeiten die mit dieser Methode gewonnenen Ergebnisse nicht mehr.)

6) dass die Symmetrieebene des granen, nachträglich aufgehellten Saumes der Unterseite des Froscheies meist mit der ersten Furchungsebene zusammenfüllt (Archir für mikr. Anat. Bd. 55. pag. 176);

7) dass die Pigmentstraße des Samenkörpers im Ei in oder dicht neben der Medianebene liegt (l. c. pag. 183);

5) dass Froschembryonen mit dem Medullarrohr nach unten liegen künnen, wie er jetzt endlich auch gesehen hat.

Zuletzt findet es 0 . Schcltze züberflitssig", dass ich seine erste Ableitung der von ihm erhaltenen Doppelbildungen ron einer Theilung des Schwerpunktes erwähnt habe, obschon er danach eine andere Ableitung vertreten habe, welche sich, wie auch die spätere Ableitung WETZEL's, mehr an die sogleich in der Diskussion von mir gegebene Ableitung anchließt, ohne dass diese Autoren sich dessen bewusst zu sein scheinen.

Die Auffrischung geschah, weil diese Ableitung zusammen mit Schultze's Auffassung ron der absoluten Nothwendigkeit der normalen Schwerkraftwirkung zur Entwickelung und mit seinem Nichtverstehen der Bedentung meiner Rotationsversuche ein seine physikalisehen Vorstellumgen sehr bezeichnendes Bild giebt. Hoffentlich wird er später die beiden letzteren seiner Auffassungen einst ebenso zurickweisen, wie jetzt die erstere.

Es wäre aber in Bezug auf diese angemessener gewesen, wenn er die Wiedererwähnung der angeblichen »Entstehung der Doppelbildungen durch Theilung des Schwerpunktes « nicht desshalb zurïckgewiesen hätte, weil er eine andere Erklärung acceptirt hat, sondern weil er eingesehen habe, dass sie eine physikalische Unmöglichkeit ist. 